\title{
Slow light of dark pulses in a photorefractive crystal
}

\author{
Nacera Bouldja $\odot,{ }^{1,2, *}$ Alexander Grabar $\odot,{ }^{3}$ Marc Sciamanna, ${ }^{1,2}$ and Delphine Wolfersberger ${ }^{1,2}$ \\ ${ }^{1}$ Chaire Photonique, LMOPS-EA 4423 and Centrale Supélec, F-57 070, Metz, France \\ ${ }^{2}$ Université de Lorraine, LMOPS-EA 4423, F-57070 Metz, France \\ ${ }^{3}$ Institute of Solid State Physics and Chemistry, Uzhhorod National University, Pidhirna 46,88000 Uzhhorod, Ukraine
}

(Received 10 April 2020; accepted 26 June 2020; published 21 July 2020)

\begin{abstract}
Slowdown of Gaussian "dark pulses" in photorefractive crystal using a two-wave mixing method is shown at room temperature. In agreement with our theoretical model, the experiment shows the dependence of the dark pulse delay and the group velocity values on the photorefractive gain $\Gamma$ and the input pulse width $t_{0}$. The use of dark pulses allows us to improve the time-delay values and achieve maximal fractional of about 1 for a pulse duration close to the crystal response time.
\end{abstract}

DOI: 10.1103/PhysRevResearch.2.032022

Slow light in dispersive nonlinear media was actively studied theoretically and experimentally [1-5], including in photorefractive (PR) materials [6-11]. The two-wave mixing (TWM) process in PR crystal yields amplification of the output pulse during the recording of the photorefractive grating. Simultaneously this modifies the light dispersion inside the crystal, hence reducing the group velocity of the output amplified pulse down to $1 \mathrm{~cm} / \mathrm{s}$ in $\mathrm{BaTiO}_{3}$ [12] and $\mathrm{Sn}_{2} \mathrm{P}_{2} \mathrm{~S}_{6}$ [10] at room temperature. The four-wave mixing effect can also be used to decelerate the light velocity both of the transmitted and the phase conjugate waves [13]. The output pulse delays vary as a function of the PR nonlinearity [14], the crystal response time, the input pulse duration [7], and the photorefractive gain $\Gamma[10]$. Small values of group velocity (large delays) are achieved for large $\Gamma$ values. However, the dispersion inside the crystal limits the delay bandwidth product because of the broadening of the output pulse.

Previous studies of slow light used Gaussian pulses because it is better suited to numerical calculations and easier to process than other pulse shapes. However, experimental and numerical studies have shown that the dark pulse or "Gaussian holes" can be more stable and less sensitive to noise in optical fibers than the Gaussian light pulses. For example, the dark soliton can be interesting for long-distance optical communications [15] due to its stable propagation in optical fibers under the influence of noise. Since their first observation [16], the dark pulses have attracted great interest, particularly for studying the propagation of solitons in the optical fiber $[17,18]$ and in Bose Einstein condensate [19]. Dark pulses arise in many distinct areas of physics: Coherent quantum control of two-photon transitions in caesium (Cs) [20], timeresolved x-ray spectroscopy [21], generation of Kerr combs

\footnotetext{
*nacera.bouldja@centralesupelec.fr

Published by the American Physical Society under the terms of the Creative Commons Attribution 4.0 International license. Further distribution of this work must maintain attribution to the author(s) and the published article's title, journal citation, and DOI.
}

in normal-dispersion microresonators with mode-interactionassisted excitation, and mode-locking transitions [22]. The use of dark pulses has been recently suggested in slow-light systems including in the plasmas [23] and insulator-insulatormetal plasmonic waveguides [24]. It has been analytically and numerically demonstrated that the dispersion of the output signal can be compensated by using Kerr dielectrics and dark solitons, and hence it of interest for high-bit-rate telecommunications [24].

In this work, we investigate theoretically and experimentally how dark pulses may be decelerating in a PR crystal at room temperature. First, we developed an analytical model describing the temporal envelope of the dark pulse propagating through the PR crystal. As for the Gaussian light pulses, the model shows that the delay and shape of the output dark pulse change as a function of the TWM gain and the input pulse duration. Using the TWM between the dark pulse and a continuous pump beam, delays greater than the input pulse duration can be reached. For $m s$ pulse duration, the corresponding delays are larger than those we have reported in Ref. [10] for Gaussian pulses and the fractional delay is also larger close to 1 . To analyze the delay performances of slow dark pulse in the PR material, the numerical calculations are implemented using the experimental parameters for a Tedoped $\mathrm{Sn}_{2} \mathrm{P}_{2} \mathrm{~S}_{6}$ crystal. Let us consider the input dark pulse $A(0, t)$ as the following:

$$
A(0, t)=\sqrt{A_{0}^{2}-A_{0}^{2} \exp \left[-\left(\frac{t}{t_{1}}\right)^{2}\right]} .
$$

Here $A_{0}$ and $t_{1}$ are respectively the amplitude and $1 / e$ halfwidth of the input pulse intensity. Using the Taylor expansion, Eq. (1) becomes

$$
A(0, t)=A_{0}\left(1-\sum_{n=0}^{\infty} a_{n} \exp \left[-2 n\left(\frac{t}{t_{1}}\right)^{2}\right]\right),
$$

where $a_{n}$ is given by

$$
a_{n}=\frac{(2 n) !}{2^{2 n}(2 n-1)(n !)^{2}} .
$$




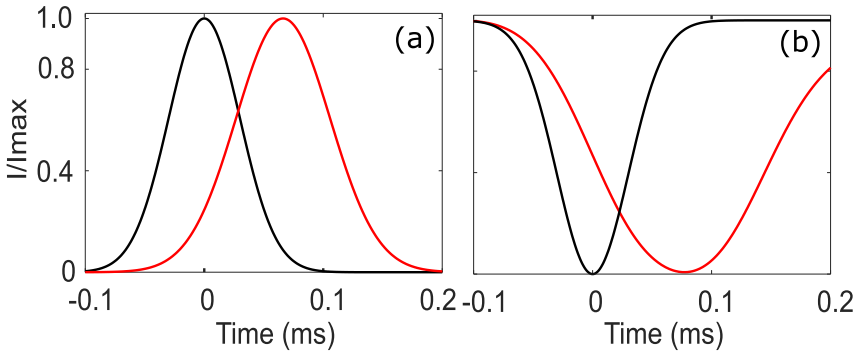

FIG. 1. Temporal envelopes of the normalized input (black line) and output (red line) pulses for input full pulse width at half maximum $t_{0}=60 \mathrm{~ms}$ and $|\Gamma d|=6.62$, (a) time delay $\Delta \tau=67 \mathrm{~ms}$ for the Gaussian light pulse and (b) $\Delta \tau=77 \mathrm{~ms}$ for the dark Gaussian pulse.

The propagation of the dark pulse in the photorefractive crystal with thickness $d$ after the interference with a continuous pump wave is expressed as follows:

$$
A(d, \omega)=A_{\omega}(0) e^{g f_{\omega}},
$$

where $A_{\omega}(0)$ is the Fourier transform of $A(0, t), g=\Gamma d$ is the dimensionless parameter conventionally referred to as the coupling strength with $\Gamma$ being the PR gain, $d$ being the crystal thickness, and $f_{\omega}$ [25] being the characteristic response function. By using the inverse Fourier transform, the output dark pulse amplitude $A(d, t)$ transmitted through the $\mathrm{PR}$ crystal is given by

$$
\begin{aligned}
A(d, t)= & A_{0} \int_{-\infty}^{\infty}\left[\delta(\omega) e^{g f_{\omega}}\right] d \omega-A_{0} \frac{t_{1}}{2 \sqrt{\pi}} \sum_{n=0}^{\infty} a_{n} \\
& \times \int_{-\infty}^{\infty} e^{-0.5 n \omega^{2} t_{1}^{2}+g f_{\omega}-i \omega t} d \omega .
\end{aligned}
$$

We use the expression of the output signal (Eq. (7) in Ref. [25]) and Eq. (5) to plot respectively the temporal envelope of bright [Fig. 1(a)] and dark [Fig. 1(b)] pulses calculated for $|\Gamma d|=6.62$ and full input width at half maximum $t_{0}=$ $60 \mathrm{~ms}$. By analyzing the profiles of the normalized pulse intensities, we can retrieve the output pulse delay $\Delta \tau$, which is the time shift between the maximum or (minimum) between the input and output light pulses or (dark pulses). The result shows that for the same $t_{0}$ the minimum of the output dark pulse [Fig. 1(b)] is more delayed than to the output bright pulse maximum [Fig. 1(a)]. For $t_{0}=60 \mathrm{~ms}$, the bright and the dark pulses are respectively delayed by $\Delta \tau=67 \mathrm{~ms}$ and $\Delta \tau=77 \mathrm{~ms}$ for $\Gamma d=|6.62|$. To illustrate the dependence of the time delay versus the TWM gain $\Gamma$, we plot in Fig. 2 the temporal profile of the input and output intensities for $t_{0}=$ $60 \mathrm{~ms}$ for three values of $|\Gamma d|=5,8,10$. As for the bright pulses, it is clearly seen that the dark pulse delay can be controlled by the gain. Both delay and output pulse width increase with increasing $\Gamma d$ values. For $|\Gamma d|=5,8,10$, the transmitted pulse delays are $\Delta \tau=50,80,100 \mathrm{~ms}$ respectively. Also, Figs. 1 and 2 show that the transmitted pulse broadens when propagating through the PR crystal. This broadening increases with the increase of the PR gain value. For $|\Gamma d|=5,8$, and 10 , the durations of the output pulse become 120,130, and $150 \mathrm{~ms}$ respectively.

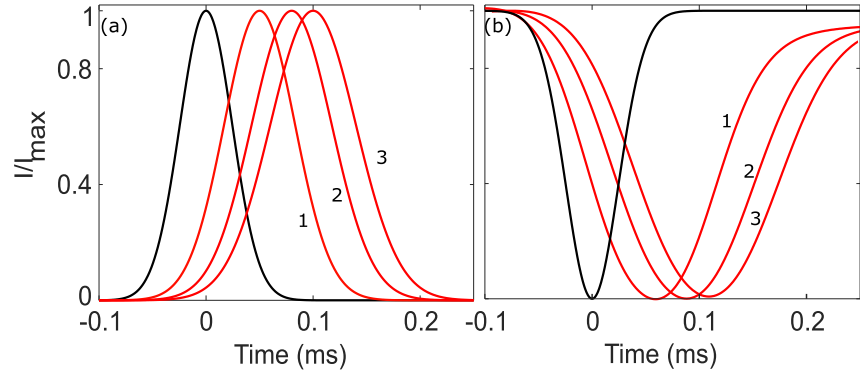

FIG. 2. Temporal envelopes of the normalized input (black line) and output (red line) pulses for input pulse duration $t_{0}=60 \mathrm{~ms}$. (a) Time delay $\Delta \tau=50,80,100 \mathrm{~ms}$ for the Gaussian light pulse and (b) $\Delta \tau=58,88,106 \mathrm{~ms}$ for the dark Gaussian pulse (1) $|\Gamma d|=5$, (2) $|\Gamma d|=8$, and $|\Gamma d|=10$.

Below we present the results of experimental studies of the slow dark pulses using the setup presented in Fig. 3. The setup is similar to that used in Ref. [10]. The measurements are performed with a laser at $\lambda=638 \mathrm{~nm}$ with intensity of $3.8 \mathrm{~W} / \mathrm{cm}^{2}$ and a 5 -mm-thick $\mathrm{Sn}_{2} \mathrm{P}_{2} \mathrm{~S}_{6}$ :Te PR crystal. The electro-optic modulator which is controlled by a signal generator generates bright or dark pulses by a change of the electric signal phase (the phase difference between a dark and bright pulse is $180^{\circ}$ ).

With this setup, we study first the experimental performances of the slow dark pulses in $\mathrm{Sn}_{2} \mathrm{P}_{2} \mathrm{~S}_{6}$ for the full input pulse duration at half maximum $t_{0}=10 \mathrm{~ms}$ as a function of the TWM gain. To measure $\Gamma$, we change the pump and signal ratio and we measure the amplification of the output signal which gives the value of the gain. The results are shown in Fig. 4. Figure 4(a) presents the normalized input and output pulse intensities for two experimental values of the gain $\Gamma d=2$ and $\Gamma d=2.9$. As is shown, the delay $\Delta \tau$ between the minimum of the input and output pulse changes with $\Gamma d$. The transmitted pulse propagates in the crystal without any broadening for $\Gamma d=2$ and when $\Gamma d$ increases to 4.6, the output pulse duration $t_{\text {out }(F H W M)}$ changes from 10 to $28 \mathrm{~ms}$. As is presented in Fig. 4(b), beyond $\Gamma d=2.4$, the measured delay $\Delta \tau$ is larger than the dark pulse duration $t_{0}$ and a maximum value of $16.5 \mathrm{~ms}$ is measured for $\Gamma d=4.6$, which is larger than the delay reported in Ref. [10] for the same input pulse duration. Figure 4(c) shows the variation of

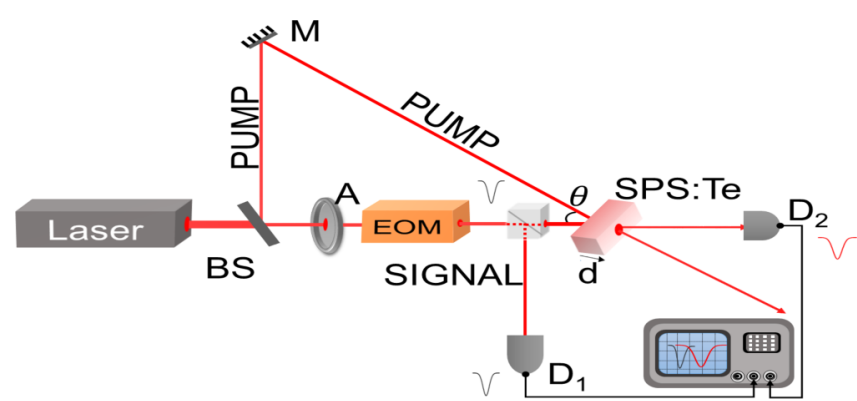

FIG. 3. Experimental setup: BS is the beam splitter, A is the attenuator, EOM is the electrooptic modulator, $D_{1,2}$ are the detectors, $\mathrm{M}$ is the mirror, $\theta$ is the incidence angle, and $\mathrm{d}$ is the crystal thickness. 

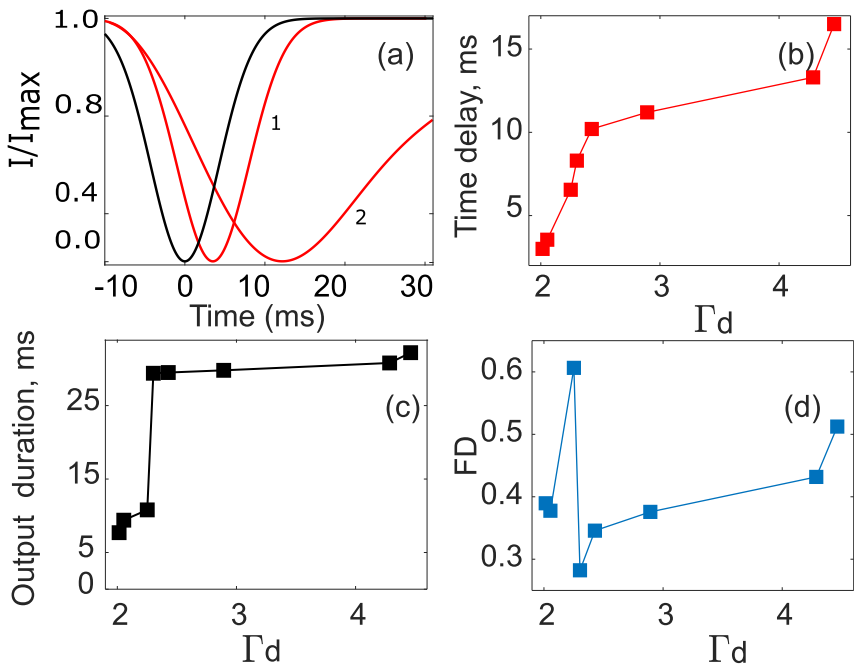

FIG. 4. Performances of the slow dark pulse as function of $\Gamma d$. (a) Temporal envelopes of the normalized input (black line) and output (red line) pulses for input pulse duration $t_{0}=10 \mathrm{~ms}$ and $\Gamma d=2$ (1) and $\Gamma d=2.9$ (2). Panels (b), (c), and (d) respectively: Time delay $\Delta \tau$, output pulse duration $t_{\text {out }(F H W M)}$, and fractional delay FD as function of $\Gamma d$.

the output pulse duration as a function of $\Gamma d$. Note that the output pulse broadening increases weakly for small values of $\Gamma d$ and becomes stronger for $\Gamma d>2.9$. Figure $4(\mathrm{~d})$ shows the measured fractional delay (FD), which is the ratio of the delay to the output full pulse width at half maximum, as function of $\Gamma d$. We see that FD varies from 0.3 to 0.6. It increases with the delay until reaching the maximum at
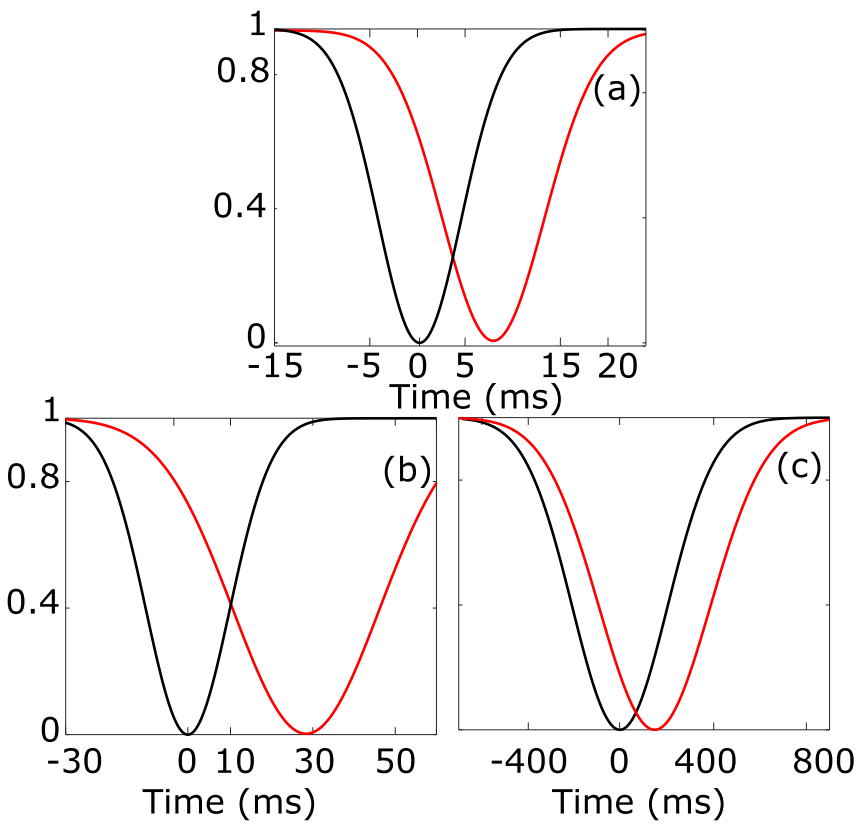

FIG. 5. Temporal envelopes of the normalized input (black line) and output (red line) pulses for $\Gamma d=5 \mathrm{~cm}^{-1}$ : (a) time delay $\Delta \tau=$ $7.9 \mathrm{~ms}, t_{0}=10.3 \mathrm{~ms}$, and $t_{\text {out }(F H W M)}=15.7 \mathrm{~ms}$, (b) $\Delta \tau=28 \mathrm{~ms}$, $t_{0}=23.5 \mathrm{~ms}$, and $t_{\text {out }(F H W M)}=56.8 \mathrm{~ms}$, and $\Delta \tau=146 \mathrm{~ms}, t_{0}=$ $480 \mathrm{~ms}$, and $t_{\text {out }(F H W M)}=570 \mathrm{~ms}$.

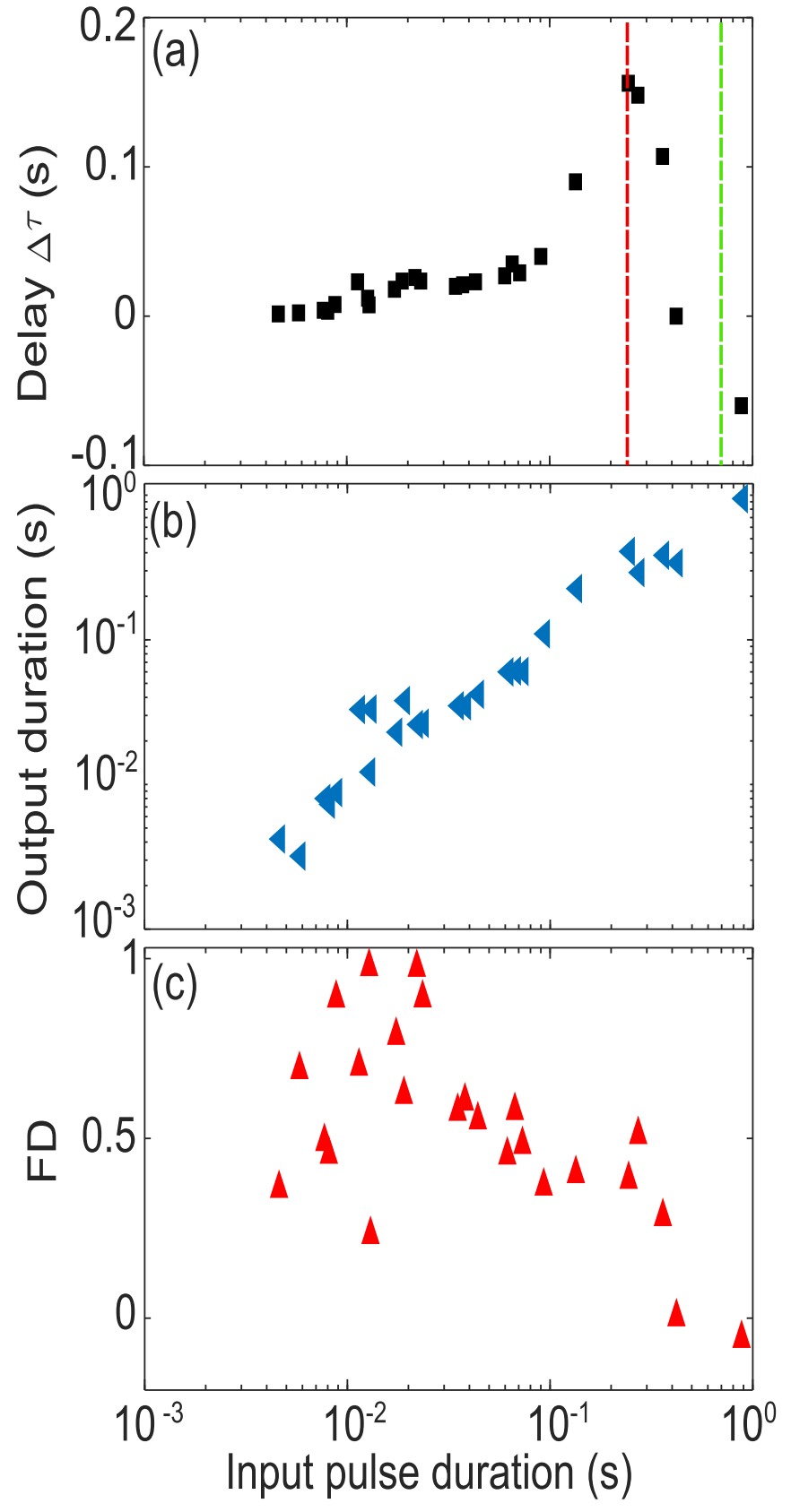

FIG. 6. Performances of the slow dark pulses according to the input pulse duration $t_{0}$ and $\Gamma d=5$. (a) Delay curve, (b) output pulse durations, and (c) fractional delay. Dotted red line presents the position of the maximum delay. Dotted green line presents the position of negative delay values.

$\Gamma d=2.4$ and decreases with the enlargement of the output pulse.

Now, as reported in Ref. [10], we analyze how the delay varies with the input duration for large TWM $\Gamma d=5$. This $\Gamma d$ value is achieved for a very weak dark pulse intensity, i.e., the ratio between the pump and the signal is of order $7 \times 10^{3}$. The curves $(\mathrm{ac})$ in Fig. 5 show the profiles of the normalized input and output pulse intensities for three input pulse durations. For example, at $t_{0}=10.3 \mathrm{~ms}$ close to the crystal response time $\tau=10 \mathrm{~ms}$, the output pulse of duration $t_{\text {out }}=15.7 \mathrm{~ms}$ is delayed by $\Delta \tau=7.9 \mathrm{~ms}$. Figure $5(\mathrm{~b})$ 
presents a case where the delay $\Delta \tau=28 \mathrm{~ms}$ is greater than the input pulse duration $t_{0}=23.5 \mathrm{~ms}$. If we increase the input pulse duration, the delay increases with $t_{0}$, and a delay of the order of $146 \mathrm{~ms}$ is measured for a pulse duration of order of $480 \mathrm{~ms}$ [Fig. 5(c)]. Therefore, there is a range of input pulse durations for which the delay reaches a maximum value relatively to $t_{0}$.

The variation of the delay, the output pulse duration, and the corresponding FD with respect to the input pulse durations are plotted in Fig. 6. Figure 6(a) shows that delay values increase for the short input pulses durations $t_{0}<250 \mathrm{~ms}$ decreasing to zero. A maximum delay value of order of $156 \mathrm{~ms}$ corresponding to the envelop group velocity $v_{g}=3.2 \mathrm{~cm} / \mathrm{s}$ is measured for input pulse duration $t_{0}=240 \mathrm{~ms}$. Also, for pulse durations higher than $880 \mathrm{~ms}$, the delay values become negative which cause the acceleration of the output dark pulse. This case is possible because of the $\mathrm{Sn}_{2} \mathrm{P}_{2} \mathrm{~S}_{6}$ selfcompensation in PR response [9]. The light acceleration can be observed by rotating the $c$ axis of the crystal by $180^{\circ}$ or by increasing the input pulse duration $t_{0}>\tau$. In our case, the advance time is measured for a pulse duration greater than $800 \mathrm{~ms}$. The output dark pulse time width versus the input pulses durations are plotted in Fig. 6(b). We note the enlargement of the output pulse duration for higher $\Gamma d$ values. For example, the input pulse of $t_{0}=19 \mathrm{~ms}$ is slowed down by $\Delta \tau=23 \mathrm{~ms}$ and its duration is almost doubled at the output of the crystal. Knowing the output pulse duration and the pulse delay, we can compute in Fig. 6(c) the fractional delay. Figure 6(c) shows that the FD is maximum for a given input pulse duration $t_{0}$ which is close to the response time of the crystal $\tau$. When $t_{0} \ll \tau$ and increasing $t_{0}$, the delay remains quite constant while the broadening of the output pulse increases, hence decreasing FD. When $t_{0} \gg \tau$ and increasing $t_{0}$, the delay increases much slowly than the increase of the broadening of the pulse, hence also decreasing FD. An optimal value of FD is therefore achieved for an intermediate value of $t_{0}$ close to $\tau$.

In summary, we have studied the slowdown of dark pulses using TWM in the $\mathrm{Sn}_{2} \mathrm{P}_{2} \mathrm{~S}_{6}$ :Te photorefractive crystal at room temperature. In addition to the experiments, the numerical calculations illustrate the performances of pulses deceleration including the time delay, the resulting distortion, and the fractional delay. The results show that the delay increases with the input duration and also with the nonlinear gain $\Gamma d$. The experimental results confirm the validity of the theoretical model. It is shown that the dark pulse is more delayed than bright pulse [10] for input pulse duration in the range $0<$ $t_{0}<100 \mathrm{~ms}$. A maximum time delay of $156 \mathrm{~ms}$ is achieved for input dark duration $t_{0}=240 \mathrm{~ms}$, corresponding to a group velocity of $v_{g}=3.2 \mathrm{~cm} / \mathrm{s}$. Increasing $\Gamma d$ leads to longer delay but the dark output pulse also propagates in the PR crystal with a larger distortion. Finally, the fractional delays 1 have been obtained for input pulse duration $t_{0}$ around the crystal PR response time $t_{r}=10 \mathrm{~ms}$, a situation for which the delay $\Delta \tau$ is larger than $t_{0}$.

Acknowledgments. Chair in Photonics is supported by Region Grand Est, Airbus GDI Simulation, Département de la Moselle, European Regional Development Fund (ERDF), CentraleSupeléc, Fondation Supeléc, Metz Métropole. A.G. is thankful for kind invitation and hospitality during his visit to CentralSupelec-LMOPS as invited professor.
[1] L. Thévenaz, Slow and fast light in optical fibres, Nat. Photo. 2 , 474 (2008).

[2] T. Baba, Slow light in photonic crystals, Nat. Photon. 2, 465 (2008).

[3] Y. Okawachi, M. S. Bigelow, J. E. Sharping, Z. Zhu, A. Schweinsberg, D. J. Gauthier, R. W. Boyd, and A. L. Gaeta, Tunable All-Optical Delays Via Brillouin Slow Light in an Optical Fiber, Phys. Rev. Lett. 94, 153902 (2005).

[4] B. Corcoran, C. Monat, C. Grillet, D. J. Moss, B. J. Eggleton, T. P. White, L. O'Faolain, and T. F. Krauss, Green light emission in silicon through slow-light enhanced third-harmonic generation in photonic-crystal waveguides, Nat. Photon. 3, 206 (2009).

[5] S. Residori, U. Bortolozzo, and J.-P. Huignard, Slow and Fast Light in Liquid Crystal Light Valves, Phys. Rev. Lett. 100, 203603 (2008).

[6] N. Bouldja, M. Sciamanna, and D. Wolfersberger, Slow light with photorefractive beam fanning, Opt. Express 28, 5860 (2020).

[7] A. Grabar, P. Mathey, and G. Gadret, Manipulation of fast light using photorefractive beam fanning, J. Opt. Soc. Am. B 31, 980 (2014).

[8] G. Zhang, R. Dong, F. Bo, and J. Xu, Slowdown of group velocity of light by means of phase coupling in photorefractive two-wave mixing, Appl. Opt. 43, 1167 (2004).
[9] A. Shumelyuk and S. Odoulov, Light pulse manipulation in $\mathrm{Sn}_{2} \mathrm{P}_{2} \mathrm{~S}_{6}$, J. Opt. 12, 104015 (2010).

[10] N. Bouldja, M. Sciamanna, and D. Wolfersberger, Improved slow light performances using photorefractive two-wave mixing, Opt. Lett. 44, 1496 (2019).

[11] G. Zhang, F. Bo, R. Dong, and J. Xu, Phase-Coupling-Induced Ultraslow Light Propagation in Solids at Room Temperature, Phys. Rev. Lett. 93, 133903 (2004).

[12] E. Podivilov, B. Sturman, A. Shumelyuk, and S. Odoulov, Light Pulse Slowing Down Up to $0.025 \mathrm{~cm} / \mathrm{s}$ by Photorefractive TwoWave Coupling, Phys. Rev. Lett. 91, 083902 (2003).

[13] K. Shcherbin, G. Gadret, H. R. Jauslin, A. Kamshilin, and P. Mathey, Slowing down of light pulses using backward-wave four-wave mixing with local response, J. Opt. Soc. Am. B 32, 2536 (2015).

[14] B. I. Sturman, E. V. Podivilov, and M. V. Gorkunov, Photorefractive manipulation of light pulses, Phys. Rev. A. 77, 063808 (2008).

[15] Y. S. Kivshar and B. Luther-Davies, Dark optical solitons: Physics and applications, Phys. Rep. 298, 81 (1998).

[16] A. Hasegawa and F. Tappert, Transmission of stationary nonlinear optical pulses in dispersive dielectric fibers. II. Normal dispersion, Appl. Phys. Lett. 23, 171 (1973).

[17] D. Krökel, N. J. Halas, G. Giuliani, and D. Grischkowsky, Dark-Pulse Propagation in Optical Fibers, Phys. Rev. Lett. 60, 29 (1988). 
[18] W.-h. Cao, S. Li, and K.-t. Chan, Generation of dark pulse trains from continuous-wave light using cross-phase modulation in optical fibers, Appl. Phys. Lett. 74, 510 (1999).

[19] D. Frantzeskakis, Dark solitons in atomic Bose-Einstein condensates: From theory to experiments, J. Phys. A: Math. Theor. 43, 213001 (2010).

[20] D. Meshulach and Y. Silberberg, Coherent quantum control of two-photon transitions by a femtosecond laser pulse, Nature (London) 396, 239 (1998).

[21] R. W. Schoenlein, S. Chattopadhyay, H. H. W. Chong, T. E. Glover, P. A. Heimann, C. V. Shank, A. A. Zholents, and M. S. Zolotorev, Generation of femtosecond pulses of synchrotron radiation, Science 287, 2237 (2000).
[22] X. Xue, Y. Xuan, Y. Liu, P.-H. Wang, S. Chen, J. Wang, D. E. Leaird, M. Qi, and A. M. Weiner, Mode-locked dark pulse Kerr combs in normal-dispersion microresonators, Nat. Photon. 9. 594 (2015).

[23] D. Farina and S. Bulanov, Slow electromagnetic solitons in electron-ion plasmas, Plasma Phys. Rep. 27, 641 (2001).

[24] E. Fitrakis, T. Kamalakis, and T. Sphicopoulos, Slow-light dark solitons in insulator-insulator-metal plasmonic waveguides, J. Opt. Soc. Am. B 27, 1701 (2010).

[25] B. Sturman, P. Mathey, and H.-R. Jauslin, Slowdown and speedup of light pulses using the self-compensating photorefractive response, J. Opt. Soc. Am. B 28, 347 (2011). 